\title{
First Characterization of a New Method for Numerically Solving the Dirichlet Problem of the Two-Dimensional Electrical Impedance Equation
}

\author{
Marco Pedro Ramirez-Tachiquin, ${ }^{1}$ Cesar Marco Antonio Robles Gonzalez, ${ }^{2}$ \\ Rogelio Adrian Hernandez-Becerril, ${ }^{2}$ and Ariana Guadalupe Bucio Ramirez \\ ${ }^{1}$ Communications and Digital Signal Processing Group, Faculty of Engineering, La Salle University, B. Franklin 47, Mexico City 06140, \\ Mexico \\ ${ }^{2}$ SEPI, ESIME Culhuacan, National Polytechnic Institute, Avenue Santa Ana No. 1000, Mexico City 04430, Mexico \\ ${ }^{3}$ SEPI, UPIITA, National Polytechnic Institute, Avenue IPN 2580, Mexico City 07340, Mexico
}

Correspondence should be addressed to Marco Pedro Ramirez-Tachiquin; marco.ramirez@lasallistas.org.mx

Received 13 March 2013; Accepted 31 May 2013

Academic Editor: Yansheng Liu

Copyright (C) 2013 Marco Pedro Ramirez-Tachiquin et al. This is an open access article distributed under the Creative Commons Attribution License, which permits unrestricted use, distribution, and reproduction in any medium, provided the original work is properly cited.

\begin{abstract}
Based upon the elements of the modern pseudoanalytic function theory, we analyze a new method for numerically solving the forward Dirichlet boundary value problem corresponding to the two-dimensional electrical impedance equation. The analysis is performed by introducing interpolating piecewise separable-variables conductivity functions in the unit circle. To warrant the effectiveness of the posed method, we consider several examples of conductivity functions, whose boundary conditions are exact solutions of the electrical impedance equation, performing a brief comparison with the finite element method. Finally, we discuss the possible contributions of these results to the field of the electrical impedance tomography.
\end{abstract}

\section{Introduction}

The study of the forward Dirichlet boundary value problem for the electrical impedance equation in the plane,

$$
\operatorname{div}(\sigma \operatorname{grad} u)=0,
$$

is fundamental for understanding its inverse problem, commonly known as electrical impedance tomography, first correctly posed in mathematical form by Calderon [1] in 1980. It is remarkable that, for more than twenty years after the problem was stated, the mathematical complexity of (1) could provoke that many experts considered impossible to obtain its general solution in analytic form [2], even for the simplest cases of $\sigma$, excluding the constant case.

This perception changed when, independently, Kravchenko in 2005 [3] and Astala and Päivärinta [4] in 2006 noticed that the two-dimensional case of (1) was completely equivalent to a special class of Vekua equation [5].
Many other important results were obtained soon after. Indeed, Kravchenko et al. published in 2007 what can be considered the first general solution of (1) in analytic form [6], when $\sigma$ possesses a certain form, employing Taylor series in formal powers [7]. Moreover, when the conductivity is separable variables, the real parts of the formal powers conform a complete set for approaching solutions of the forward Dirichlet boundary value problem of (1) in the plane [8].

The main objective of this work is to start a discussion about the application of the Pseudoanalytic function theory when $\sigma$ is not, in general, a separable-variables function, which would allow the study of conductivity cases more interesting in physics and engineering. As a matter of fact, even it is not clear yet how to extend the proof provided in [8], about the completeness of the set of formal powers for the cases when $\sigma$ is piecewise separable variables, the numerical calculations will show that a variation of the technique, 
originally posed for purely mathematical problems, could well serve for analyzing more general cases, providing quite acceptable results with compared when classical methods.

In other words, this work states that if the values of the electrical conductivity are known at every point within a bounded domain $\Omega$ in the plane, it will be always possible to introduce a piecewise separable-variables function, such that we can use it to obtain a set of base functions for approaching solutions of the forward Dirichlet boundary value problem of (1), employing pseudoanalytic functions. This would be true for a certain class of bounded domains, but this class will be wide enough to include many interesting cases for the applied sciences. Nonetheless, on behalf of briefness, we will only study the unit circle. The reader will appreciate that most of the results will be valid for more domains.

In order to prove the veracity of the last assessments, we will consider a set of conductivity examples, separable variables, and nonseparable variables, for which we can obtain exact solutions, to be imposed as boundary conditions. The effectiveness of the approaches will be estimated by comparing them with the boundary conditions, employing a standard Lebesgue measure.

Finally, we will discuss how this numerical technique could contribute to the study of the inverse Dirichlet boundary value problem of (1) in the plane, also known as the Electrical Impedance Tomography problem.

\section{Preliminaries}

According to the Pseudoanalytic function theory posed by Bers [7], let the pair of complex-valued functions $F$ and $G$ fulfill the condition:

$$
\operatorname{Im}(\bar{F} G)>0,
$$

where $\bar{F}$ represents the complex conjugation of $F: \bar{F}=\operatorname{Re} F-$ $i \operatorname{Im} F$ and $i$ denotes the standard imaginary unit $i^{2}=-1$. This condition implies that the functions $F$ and $G$ are linearly independent, therefore any complex-valued function $W$ can be expressed by their linear combination:

$$
W=\phi F+\psi G,
$$

where $\phi$ and $\psi$ are purely real-valued functions. On the light of this idea, L. Bers introduced the concept of the $(F, G)$ derivative of $W$ in the form

$$
\partial_{(F, G)} W=\left(\partial_{z} \phi\right) F+\left(\partial_{z} \psi\right) G .
$$

This derivative will exist if and only if the following equality is true:

$$
\left(\partial_{\bar{z}} \phi\right) F+\left(\partial_{\bar{z}} \psi\right) G=0
$$

Hereafter, we will use the notations $\partial_{z}=\partial_{x}-i \partial_{y}$ and $\partial_{\bar{z}}=$ $\partial_{x}+i \partial_{y}$. These pairs of partial differential operators are usually introduced with the factor $1 / 2$, but in this work it will be somehow more convenient to work without it.
Introducing the notations:

$$
\begin{aligned}
A_{(F, G)}=\frac{\bar{F} \partial_{z} G-\bar{G} \partial_{z} F}{F \bar{G}-G \bar{F}}, & a_{(F, G)}=-\frac{\bar{F} \partial_{\bar{z}} G-\bar{G} \partial_{\bar{z}} F}{F \bar{G}-G \bar{F}}, \\
B_{(F, G)}=\frac{F \partial_{z} G-G \partial_{z} F}{F \bar{G}-G \bar{F}}, & b_{(F, G)}=-\frac{G \partial_{\bar{z}} F-F \partial_{\bar{z}} G}{F \bar{G}-G \bar{F}},
\end{aligned}
$$

the derivative presented in (4), that we will refer to as the $(F, G)$-derivative of $W$, can be rewritten as

$$
\partial_{(F, G)} W=\partial_{z} W-A_{(F, G)} W-B_{(F, G)} \bar{W}
$$

whereas condition (5) will become

$$
\partial_{\bar{z}} W-a_{(F, G)} W-b_{(F, G)} \bar{W}=0 .
$$

A pair of complex functions $(F, G)$ satisfying condition (2) will be called a generating pair, and the functions introduced in (6) will be referred to as the characteristic coefficients of the generating pair $(F, G)$. As a matter of fact, the expression (8) is known as the Vekua equation [5], and every function $W$, solution of (8), will be called $(F, G)$ pseudoanalytic.

The following statements were originally posed in [7]. We present them with certain modifications, in order to better analyze the special class of Vekua equation corresponding to the electrical impedance equation (1) in the plane.

Theorem 1. The functions $F$ and $G$, constituting the generating pair of the form (2), are (F, G)-pseudoanalytic, and their $(F, G)$ derivatives vanish identically:

$$
\partial_{(F, G)} F \equiv \partial_{(F, G)} G \equiv 0 .
$$

Theorem 2 (see $[7,9])$. Let $p$ be a nonvanishing function, defined within some domain $\Omega\left(\mathbb{R}^{2}\right)$, and let

$$
F=p, \quad G=\frac{i}{p} .
$$

It is easy to verify that $F$ and $G$ conform a generating pair (2), whose characteristic coefficients, according to (6), are

$$
\begin{gathered}
A_{(F, G)}=a_{(F, G)}=0, \\
B_{(F, G)}=\frac{\partial_{z} p}{p}, \\
b_{(F, G)}=\frac{\partial_{\bar{z}} p}{p} .
\end{gathered}
$$

Therefore, for this special class of generating pairs, the corresponding Vekua equations will have the form

$$
\partial_{\bar{z}} W-\frac{\partial_{\bar{z}} p}{p} \bar{W}=0 .
$$

Definition 3. Let $\left(F_{0}, G_{0}\right)$ and $\left(F_{1}, G_{1}\right)$ be two generating pairs of the form (10), and let their characteristic coefficients fulfill the condition

$$
B_{\left(F_{0}, G_{0}\right)}=-b_{\left(F_{1}, G_{1}\right)} .
$$

Thus the generating pair $\left(F_{1}, G_{1}\right)$ will be called a successor pair of $\left(F_{0}, G_{0}\right)$, as well $\left(F_{0}, G_{0}\right)$ will be called a predecessor of $\left(F_{1}, G_{1}\right)$. 
Definition 4. Let the elements of the set

$$
\left\{\left(F_{m}, G_{m}\right)\right\}, \quad m=0, \pm 1, \pm 2, \ldots,
$$

be all generating pairs, and let every $\left(F_{m+1}, G_{m+1}\right)$ be a successor of $\left(F_{m}, G_{m}\right)$. Hence, the set (14) will be called a generating sequence. If $(F, G)=\left(F_{0}, G_{0}\right)$, we will say that $(F, G)$ is embedded into (14). Moreover, if there exists a number $k$ such that $\left(F_{m+k}, G_{m+k}\right)=\left(F_{m}, G_{m}\right)$, we will say that the generating sequence (14) is periodic, with period $k$.

L. Bers also introduced the concept of the $(F, G)$-integral of a complex function $W$. The reader can find the conditions for warranting its existence and a detailed description of its properties in $[7,9]$. Since the functions employed in this work are, by definition, $(F, G)$-integrable, we will only present a certain set of those properties.

Definition 5. Let $\left(F_{0}, G_{0}\right)$ be a generating pair with the form (10). Its adjoin pair $\left(F_{0}^{*}, G_{0}^{*}\right)$ is defined as

$$
F_{0}^{*}=-i F_{0}, \quad G_{0}^{*}=-i G_{0} .
$$

Definition 6. The $\left(F_{0}, G_{0}\right)$-integral of a complex function $W$ (when it exists) is defined according to the expression

$$
\int_{\Lambda} W d_{\left(F_{0}, G_{0}\right)} z=F_{0} \operatorname{Re} \int_{\Lambda} G_{0}^{*} W d z+G_{0} \operatorname{Re} \int_{\Lambda} F_{0}^{*} W d z,
$$

where $\Lambda$ is a rectifiable curve going from $z_{0}$ up to $z_{1}$, in the complex plain. In particular, the $\left(F_{0}, G_{0}\right)$-integral of $\partial_{\left(F_{0}, G_{0}\right)} W$ reaches

$$
\int_{z_{0}}^{z} \partial_{\left(F_{0}, G_{0}\right)} W d_{F_{0}, G_{0}} z=W-\phi\left(z_{0}\right) F_{0}-\psi\left(z_{0}\right) G_{0} .
$$

But according to Theorem 1, the $\left(F_{0}, G_{0}\right)$-derivatives of $F_{0}$ and $G_{0}$ vanish identically, hence (17) can be considered the $\left(F_{0}, G_{0}\right)$-antiderivative of $\partial_{\left(F_{0}, G_{0}\right)} W$.

\subsection{Formal Powers}

Definition 7. The formal power $Z_{m}^{(0)}\left(a_{0}, z_{0} ; z\right)$ belonging to the generating pair $\left(F_{m}, G_{m}\right)$, with formal exponent 0 , complex coefficient $a_{0}$, center at $z_{0}$, and depending upon the complex variable $z=x+i y$, is defined by the expression

$$
Z_{m}^{(0)}\left(a_{0}, z_{0} ; z\right)=\lambda F_{m}+\mu G_{m},
$$

where $\lambda$ and $\mu$ are constants that fulfill the condition

$$
\lambda F_{m}\left(z_{0}\right)+\mu G_{m}\left(z_{0}\right)=a_{0} .
$$

The formal powers with higher formal exponents are defined according to the recursive formulas

$$
\left.Z_{m}^{(n)}\left(a_{n}, z_{0} ; z\right)=n \int_{z_{0}}^{z} Z_{m-1}^{(n-1)}\left(a_{n}, z_{0} ; z\right) d_{\left(F_{m}, G_{m}\right.}\right) .
$$

Notice the integral operators at the right hand side of the last equality are all $\left(F_{m}, G_{m}\right)$-antiderivatives.
Remark 8. The formal powers possess the following properties:

(1) $Z_{m}^{(n)}\left(a_{n}, z_{0} ; z\right) \rightarrow a_{n}\left(z-z_{0}\right)^{n}$ when $z \rightarrow z_{0}$.

(2) Every $Z_{m}^{(n)}\left(a_{n}, z_{0} ; z\right)$ is $\left(F_{m}, G_{m}\right)$-pseudoanalytic.

(3) If $a_{n}=a_{n}^{\prime}+i a_{n}^{\prime \prime}$, where $a_{n}^{\prime}$ and $a_{n}^{\prime \prime}$ are real constants, we will have

$Z_{m}^{(n)}\left(a_{n}, z_{0} ; z\right)=a_{n}^{\prime} Z_{m}^{(n)}\left(1, z_{0} ; z\right)+a_{n}^{\prime \prime} Z_{m}^{(n)}\left(i, z_{0} ; z\right)$.

Theorem 9. Every complex-valued function $W$, solution of the Vekua equation (8), can be expanded in terms of the so-called Taylor series in formal powers:

$$
W=\sum_{n=0}^{\infty} Z^{(n)}\left(a_{n}, z_{0} ; z\right),
$$

where the absence of the subindex " $m$ " indicates that all formal powers belong to the same generating pair.

Remark 10. Since every complex-valued function $W$, solution of (8), can be expressed in the form (22), it is possible to assert that (22) is an analytic representation of the general solution for the Vekua equation (8).

2.2. The Electrical Impedance Equation in the Plane. As it has been previously posed in several works (see, e.g., $[3,9,10]$ ), when the conductivity function $\sigma$ can be expressed in terms of a separable-variables function

$$
\sigma=\sigma_{1}(x) \sigma_{2}(y),
$$

by introducing the notations

$$
\begin{gathered}
W=\sqrt{\sigma} \partial_{x} u-i \sqrt{\sigma} \partial_{y} u, \\
p=\frac{\sqrt{\sigma_{2}}}{\sqrt{\sigma_{1}}},
\end{gathered}
$$

the two-dimensional electrical impedance equation (1) can be rewritten precisely as a Vekua equation of the form (12). Moreover, its corresponding generating pair

$$
F_{0}=p, \quad G_{0}=\frac{i}{p}
$$

is embedded into a periodic generating sequence, with period $k=2$, such that

$$
F_{m}=\frac{\sqrt{\sigma_{2}}}{\sqrt{\sigma_{1}}}, \quad G_{m}=i \frac{\sqrt{\sigma_{1}}}{\sqrt{\sigma_{2}}},
$$

when $m$ is an even number, and

$$
F_{m}=\sqrt{\sigma_{1} \sigma_{2}}, \quad G_{m}=\frac{i}{\sqrt{\sigma_{1} \sigma_{2}}},
$$

when $m$ is odd. 
Therefore, based upon the statements posed in Definition 7 , possessing a generating sequence will allow us to approach a set of formal powers:

$$
\left\{Z_{0}^{(n)}(1,0 ; z), Z_{0}^{(n)}(i, 0 ; z)\right\}_{n=0}^{N} \text {, }
$$

within a bounded domain $\Omega$, and, subsequently by virtue of Remark 8, we will be able to approach any formal power $Z_{0}^{(n)}\left(a_{n}, 0 ; z\right)$, at any point $z \in \Omega$.

Because the present work intends to contribute to the construction of a novel theory for the electrical impedance tomography problem, we will focus our attention into a classic domain $\Omega$, the unit disk with center at $z_{0}=0$.

2.3. A Complete Orthonormal System. In [8] Campos et al. posed a very important property of the formal powers.

Theorem 11 (see [8]). The set of real parts of the formal powers, with coefficients 1 and $i$, valued at the boundary $\Gamma$ of a bounded domain $\Omega$

$$
\left\{\left.\operatorname{Re} Z^{(n)}(1,0 ; z)\right|_{\Gamma},\left.\operatorname{Re} Z^{(n)}(i, 0 ; z)\right|_{\Gamma}\right\}_{n=0}^{\infty}
$$

constitutes a complete system for approaching solutions of the forward Dirichlet boundary value problem for the electrical impedance equation (1) in the plane.

That is, any boundary condition $\left.u\right|_{\Gamma}$ can be approached asymptotically by the linear combination of the elements belonging to (29):

$$
\begin{aligned}
\left.\lim _{N \rightarrow \infty} u\right|_{\Gamma}- & \left(\left.\sum_{n=0}^{N} c_{n}^{(1)} \operatorname{Re} Z^{(n)}(1,0 ; z)\right|_{\Gamma}\right. \\
& \left.+\left.c_{n}^{(i)} \operatorname{Re} Z^{(n)}(i, 0 ; z)\right|_{\Gamma}\right)=0
\end{aligned}
$$

where the coefficients $c_{n}^{(1)}$ and $c_{n}^{(i)}$ are all real constants.

Since the elements of the set (29) are, by definition, linearly independent [7], it is possible to perform a standard Gram-Schmidt orthonormalization process in order to obtain the set of functions

$$
\left\{u_{n}\right\}_{n=0}^{2 N-1}
$$

defined on the boundary $\Gamma$, such that when imposing a boundary condition $\left.u\right|_{\Gamma}$, we will have

$$
\left.u\right|_{\Gamma} \sim \sum_{n=0}^{2 N-1} \alpha_{n} u_{n}
$$

Because the set $\left\{u_{k}\right\}_{k=0}^{2 N-1}$ is orthonormal, the calculation of the coefficients $\alpha_{n}$ can be performed by several classical methods. Particularly, we will employ the scalar product

$$
\alpha_{n}=\left\langle u_{n},\left.u\right|_{\Gamma}\right\rangle
$$

2.4. A Basic Numerical Approach. Remembering that $\Gamma$ coincides with the perimeter of the unit circle, we can employ the numerical methods detailed in [11] for obtaining a system of $2 N+1$ formal powers (notice that, according to (25), $\left.\operatorname{Re} Z_{0}^{(0)}\left(i, z_{0} ; z\right)=0\right)$, defined at the boundary $\Gamma$ :

$$
\left\{\left.\operatorname{Re} Z_{0}^{(n)}(1,0 ; z)\right|_{\Gamma},\left.\operatorname{Re} Z_{0}^{(n)}(i, 0 ; z)\right|_{\Gamma}\right\}_{n=0}^{N} .
$$

More precisely, let us consider the formal powers $Z_{0}^{(n)}(1,0 ; z)$. Taking into account that the integral expressions introduced in (17) are path independent [7], we can choose the rectifiable curve $\Lambda$ to be a straight line segment, going from $z_{0}=0$ until some point of the unit circle. Thus, we can allocate $P$ points equidistantly distributed on such line, obtaining the set of complex numbers

$$
\left\{z[p]=\frac{p}{P} \cos \theta[r]+i \frac{p}{P} \sin \theta[r]\right\}_{p=0}^{P-1},
$$

where $\theta[r]$ is some angle associated to the radius. Given this set of points, and considering that the generating sequence corresponding to the pair (25) is periodic with period $k=2$, we can numerically approach the formal powers employing, for example, a variation of the trapezoidal integration method according to the expressions

$$
\begin{aligned}
Z_{0}^{(n)}(z[p]) & \\
= & (n-1) F_{0}(z[p]) \\
& \times \operatorname{Re} \sum_{q=0}^{p-1}\left(Z_{1}^{(n-1)}(z[q+1]) G_{0}^{*}(z[q+1])\right) d z[q] \\
& +(n-1) F_{0}(z[p]) \\
& \times \operatorname{Re} \sum_{q=0}^{p}\left(Z_{1}^{(n-1)}(z[q]) G_{0}^{*}(z[q])\right) d z[q] \\
& +(n-1) G_{0}(z[p]) \\
& \times \operatorname{Re} \sum_{q=0}^{p-1}\left(Z_{1}^{(n-1)}(z[q+1]) F_{0}^{*}(z[q+1])\right) d z[q] \\
& +(n-1) G_{0}(z[p]) \\
& \times \operatorname{Re} \sum_{q=0}^{p}\left(Z_{1}^{(n-1)}(z[q]) F_{0}^{*}(z[q])\right) d z[q]
\end{aligned}
$$

where $p, q=0,1, \ldots, P-1$, and

$$
d z[p]=z[p+1]-z[p] .
$$

Besides, we can introduce a set of $R$ angles $\theta[r]$, to be employed in (35), according to the formula

$$
\left\{\theta[r]=\frac{2 \pi r}{R}\right\}_{r=0}^{R-1}
$$




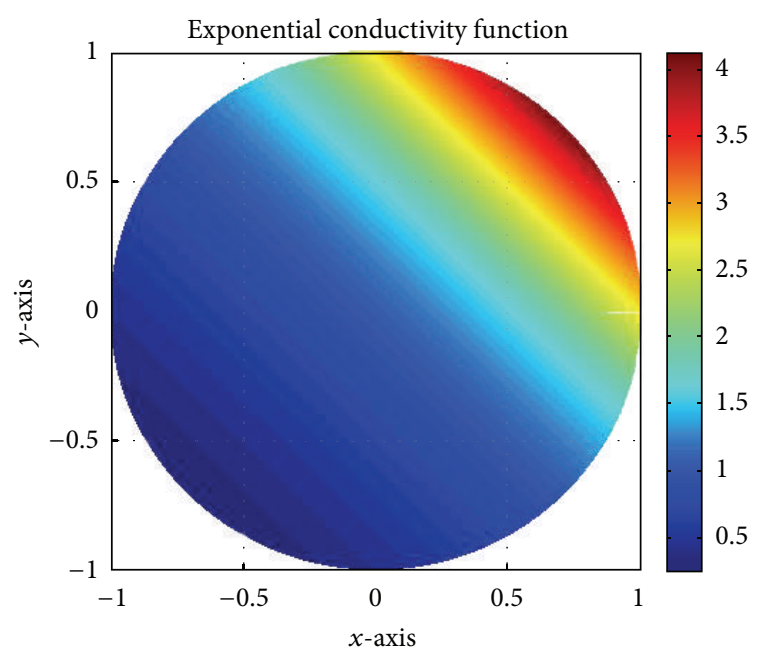

FIGURE 1: Example of a separable-variables exponential conductivity function $\sigma=e^{x+y}$.

TABLE 1: Relation between the error $\mathscr{E}$ and the number of formal powers, for the case when $\sigma$ possesses an exponential form.

\begin{tabular}{lccc}
\hline $\begin{array}{l}\text { Number of } \\
\text { radii } R\end{array}$ & $\begin{array}{c}\text { Points per } \\
\text { radius } P\end{array}$ & $\begin{array}{c}\text { Number of formal } \\
\text { powers } N\end{array}$ & $\mathscr{E}$ \\
\hline 500 & 500 & 45 & $1.4370 \times 10^{-15}$ \\
100 & 100 & 45 & $1.9996 \times 10^{-15}$ \\
100 & 100 & 35 & $2.0001 \times 10^{-15}$ \\
100 & 100 & 25 & $2.0094 \times 10^{-15}$ \\
100 & 100 & 15 & $2.0524 \times 10^{-15}$ \\
100 & 100 & 5 & $3.1333 \times 10^{-14}$ \\
\hline
\end{tabular}

for the iterative expressions (36) that can be performed at each angle $\theta[r]$. When the full procedure is complete, we will possess a set of $N+1$ formal powers $Z^{(n)}(1,0 ; z)$, approached for $R$ radii, and with $P$ points per radius. Thus, by collecting the real parts of $Z^{(n)}(1,0 ; z)$ valued at the points $z[P-1]$, which are precisely those located at the boundary $\Gamma$, we will obtain a numerical approximation of the set

$$
\left\{\left.\operatorname{Re} Z^{(n)}(1,0 ; z)\right|_{\Gamma}\right\}_{n=0}^{N}
$$

An identical procedure can be performed for obtaining the elements of

$$
\left\{\left.\operatorname{Re} Z^{(n)}(i, 0 ; z)\right|_{\Gamma}\right\}_{n=0}^{N}
$$

Since the numerical approaching of the set (34) is complete, a standard Gram-Schmidt method will reach the orthonormal system (31) for approaching the boundary condition $\left.u\right|_{\Gamma}$.

The effectiveness of this numerical approach has been analyzed in several works (see $[8,10])$. Here we will only analyze two particular examples of separable-variables conductivity functions $\sigma$, before studying those that are not separable variables.
TABLE 2: Relation between the error $\mathscr{E}$ and the number of formal powers, for the case when $\sigma$ has a Lorentzian form.

\begin{tabular}{lccc}
\hline $\begin{array}{l}\text { Number of } \\
\text { radii } R\end{array}$ & $\begin{array}{c}\text { Points per } \\
\text { radius } P\end{array}$ & $\begin{array}{c}\text { Number of formal } \\
\text { powers } N\end{array}$ & $\mathscr{E}$ \\
\hline 500 & 500 & 45 & $2.2968 \times 10^{-8}$ \\
100 & 100 & 45 & $2.3753 \times 10^{-8}$ \\
100 & 100 & 35 & $5.8069 \times 10^{-7}$ \\
100 & 100 & 25 & $1.9710 \times 10^{-5}$ \\
100 & 100 & 15 & $7.2801 \times 10^{-4}$ \\
100 & 100 & 5 & $4.8875 \times 10^{-2}$ \\
\hline
\end{tabular}

\section{The Cases When the Conductivity $\sigma$ Possesses a Separable-Variables Form}

\subsection{Example When $\sigma$ Is an Exponential Function}

Proposition 12. Let $\sigma=e^{x+y}$. Then the function $u=e^{-x-y}$ will be a particular solution of (1).

Figure 1 plots an illustration of the exponential conductivity.

The numerical procedure described in Section 2.4 will be employed for approaching the forward Dirichlet boundary value problem when $\sigma$ possesses the form of the proposition above, imposing as the boundary condition the exact solution

$$
\left.u\right|_{\Gamma}=e^{-x-y} .
$$

The error $\mathscr{E}$ will be defined according to the Lebesgue measure

$$
\mathscr{E}=\left(\int_{\Gamma}\left(\left.u\right|_{\Gamma}-\sum_{n=0}^{2 N+1} \alpha_{n} u_{n}\right)^{2} d l\right)^{1 / 2},
$$

where the addition within the integral expression corresponds to the approached solution (32).

Table 1 contains the relation between the error $\mathscr{E}$ and the number of formal powers $N$. The parameters $R$ and $P$ have been fixed at the value 100 , since for this particular case, as displayed in the table, they do not significantly influence the diminution of the error when increasing.

\subsection{The Case When $\sigma$ Has a Lorentzian Form}

Proposition 13. Let

$$
\sigma=\left(\frac{1}{x^{2}+0.1}\right)\left(\frac{1}{y^{2}+0.1}\right) .
$$

Then the function

$$
u=\frac{x^{3}+y^{3}}{3}+0.1(x+y)
$$

will be a particular solution of (1).

An illustration of the conductivity function (43) is displayed in Figure 2. 


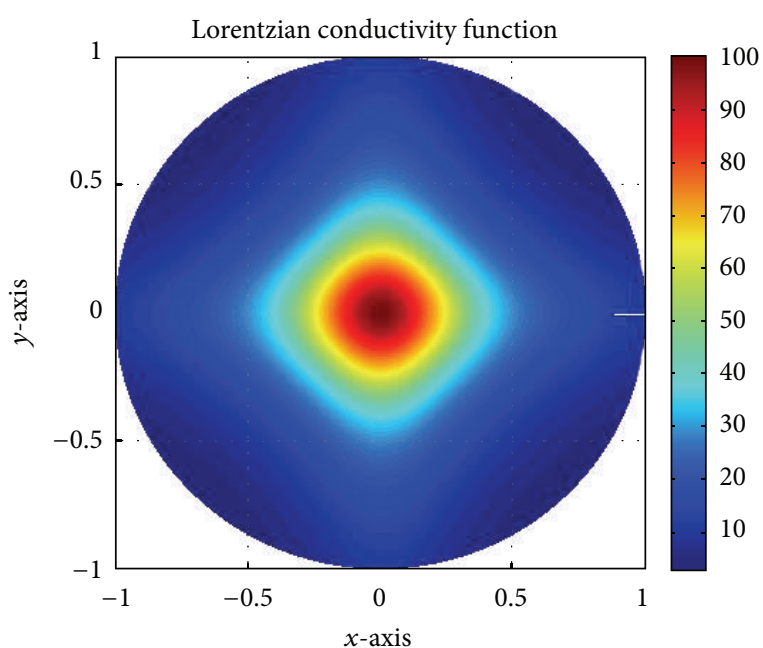

FIGURE 2: Example of a separable-variables Lorentzian conductivity function $\sigma=\left(x^{2}+0.1\right)^{-1} \cdot\left(y^{2}+0.1\right)^{-1}$.

Once more, the exact solution (44) will be imposed as the boundary condition. The numerical results are shown in Table 2. This, indeed, is a more interesting case, since the number $N$ of formal powers strongly influences the accuracy of the approach. The increment of the number of points per radius $P$ and the number of radii $R$, as in the previous case, do not significantly increase the accuracy.

\subsection{Construction of a Piecewise Separable-Variables Conduc-} tivity Function. Consider a bounded domain $\Omega\left(\mathbb{R}^{2}\right)$ (in this case the unit circle) and divide it into a finite number of subsections $S$, taking care that the point to be considered the center of the formal powers $z_{0}$ (see Definition 7) does not reside onto the boundary of two subsections. For simplicity, let us make the division by employing a finite set of parallel lines to the $x$-axis, equidistant to one another, and let us locate $z_{0}=0$.

Supposing that the values of the electrical conductivity are defined for every point within the domain $\Omega\left(\mathbb{R}^{2}\right)$, let us locate a straight line crossing every subsection, that does not intersect the bounding parallel lines of its corresponding subsection. Indeed, such lines can be simply parallel to the bounding ones.

The following step is to collect finite sets of conductivity values, corresponding to a number $C$ of points located at the crossing lines. For every line, the quantity of collected values $C$ must be big enough to warrant that an interpolating process, in this case performed with straight lines, will adequately approach all the remaining conductivity values defined over the line points.

Since we have already assumed that every crossing line will be parallel to the subsection-bounding lines, and in consequence to the $x$-axis, all collected points corresponding to the same crossing line will possess the same $y$-coordinate. Let us propose that the conductivity within every subsection can be represented according to the expression

$$
\sigma=\frac{y+K}{\Upsilon+K} \cdot f(x)
$$

where $\Upsilon$ denotes the $y$-coordinate that is common to all points along the crossing line, $f(x)$ is an interpolating function that approaches the collected conductivity values, and $K$ is a real positive constant such that $y+K \neq 0$, for all $y \in \Omega$.

Applying this idea in every subsection, the conductivity $\sigma$ within the bounded domain $\Omega$ can be approached by means of the piecewise-defined function:

$$
\sigma(x, y)=\left\{\begin{array}{l}
\frac{y+K}{\Upsilon_{1}+K} \cdot f_{1}(x): y \in\left[y_{(1)}, y_{(2)}\right) \\
\frac{y+K}{\Upsilon_{2}+K} \cdot f_{2}(x): y \in\left[y_{(2)}, y_{(3)}\right) \\
\vdots \\
\frac{y+K}{\Upsilon_{S}+K} \cdot f_{S}(x): y \in\left[y_{(S)}, y_{(S+1)}\right]
\end{array}\right.
$$

Here $y_{(1)}$ represents the first $y$-coordinate found within the domain $\Omega$ when broaching the $y$-axis from $\infty$ up to $-\infty$, whereas $y_{(S)}$ represents the last one. The pairs of $y$-axis parallel lines $\left(y_{(j)}, y_{(j+1)}\right)$, where $j=1, \ldots, S+1$, are given by the common $y$-coordinates belonging to every pair of lines delimiting the subsections. Notice the piecewise function (46) is separable variables.

Thus, according to Section 2.2, it immediately follows that

$$
F_{0}=\left\{\begin{array}{l}
\left(\frac{y+K}{\Upsilon_{1}+K} \cdot f_{1}^{-1}(x)\right)^{1 / 2}: y \in\left[y_{(1)}, y_{(2)}\right), \\
\left(\frac{y+K}{\Upsilon_{2}+K} \cdot f_{2}^{-1}(x)\right)^{1 / 2}: y \in\left[y_{(2)}, y_{(3)}\right), \\
\vdots \\
\left(\frac{y+K}{\Upsilon_{S}+K} \cdot f_{S}^{-1}(x)\right)^{1 / 2}: y \in\left[y_{(S)}, y_{(S+1)}\right],
\end{array}\right.
$$

whereas

$$
G_{0}=\left\{\begin{array}{l}
i\left(\frac{\Upsilon_{1}+K}{y+K} \cdot f_{1}(x)\right)^{1 / 2}: y \in\left[y_{(1)}, y_{(2)}\right), \\
i\left(\frac{\Upsilon_{2}+K}{y+K} \cdot f_{2}(x)\right)^{1 / 2}: y \in\left[y_{(2)}, y_{(3)}\right), \\
\vdots \\
i\left(\frac{\Upsilon_{S}+K}{y+K} \cdot f_{S}(x)\right)^{1 / 2}: y \in\left[y_{(S)}, y_{(S+1)}\right] .
\end{array}\right.
$$


TABLE 3: Relation between the error $\mathscr{E}$ and the number of sections $S$ and samples per section $C$, when $\sigma=e^{x+y}$.

\begin{tabular}{|c|c|c|c|c|c|}
\hline $\begin{array}{l}\text { Number of } \\
\text { radii } R\end{array}$ & $\begin{array}{c}\text { Points per } \\
\text { radius } P\end{array}$ & $\begin{array}{c}\text { Number of formal } \\
\text { powers } N\end{array}$ & $\begin{array}{l}\text { Number of } \\
\text { sections } S\end{array}$ & $\begin{array}{c}\text { Collected values per } \\
\text { section } C\end{array}$ & $\mathscr{E}$ \\
\hline 100 & 100 & 45 & 20001 & 20001 & $4.4935 \times 10^{-5}$ \\
\hline 100 & 100 & 45 & 10001 & 10001 & $6.9358 \times 10^{-5}$ \\
\hline 100 & 100 & 45 & 5001 & 5001 & $6.8149 \times 10^{-4}$ \\
\hline 100 & 100 & 45 & 2501 & 2501 & $5.7113 \times 10^{-4}$ \\
\hline 100 & 100 & 45 & 1001 & 1001 & $3.3381 \times 10^{-3}$ \\
\hline 100 & 100 & 45 & 501 & 501 & $1.5105 \times 10^{-3}$ \\
\hline 100 & 100 & 45 & 101 & 101 & $5.3048 \times 10^{-3}$ \\
\hline
\end{tabular}

For the generating pair $\left(F_{1}, G_{1}\right)$ we will have

$$
\begin{aligned}
& F_{1}=\left\{\begin{array}{l}
\left(\frac{y+K}{\Upsilon_{1}+K} \cdot f_{1}(x)\right)^{1 / 2}: y \in\left[y_{(1)}, y_{(2)}\right), \\
\left(\frac{y+K}{\Upsilon_{2}+K} \cdot f_{2}(x)\right)^{1 / 2}: y \in\left[y_{(2)}, y_{(3)}\right), \\
\vdots \\
\left(\frac{y+K}{\Upsilon_{S}+K} \cdot f_{S}(x)\right)^{1 / 2}: y \in\left[y_{(S)}, y_{(S+1)}\right],
\end{array}\right. \\
& G_{1}=\left\{\begin{array}{l}
i\left(\frac{\Upsilon_{1}+K}{y+K} \cdot f_{1}^{-1}(x)\right)^{1 / 2}: y \in\left[y_{(1)}, y_{(2)}\right), \\
i\left(\frac{\Upsilon_{2}+K}{y+K} \cdot f_{2}^{-1}(x)\right)^{1 / 2}: y \in\left[y_{(2)}, y_{(3)}\right), \\
\vdots \\
i\left(\frac{\Upsilon_{S}+K}{y+K} \cdot f_{S}^{-1}(x)\right)^{1 / 2}: y \in\left[y_{(S)}, y_{(S+1)}\right] .
\end{array}\right.
\end{aligned}
$$

We will use these piecewise-defined functions to perform the numerical procedure described in Section 2.4. The first case to analyze will be the exponential conductivity $\sigma=e^{x+y}$ studied in Section 3.1, imposing as the boundary condition $\left.u\right|_{\Gamma}=e^{-x-y}$ the exact solution presented in Proposition 12.

The second case will be the Lorentzian conductivity treated in Section 3.2, whose boundary condition will be the exact solution $\left.u\right|_{\Gamma}$ shown in Proposition 13. The results of these cases are exposed in Tables 3 and 4. For both, we can observe that the accuracy is strongly related with the number of sections $S$ and the number of collected values per section $C$, as well as with the number $N$ of formal powers. Once more, the increment of the number of points per radius $P$ and the number of radii $R$ do not significantly improve the accuracy.

Beside, since the tables presented in the previous subsection have shown that the accuracy of the method is not considerably improved when neither the number of points per radius $P$ nor the number of radii $R$ increase, we will fix both values $P=R=100$ hereafter.

Tables 3 and 4 show that the piecewise separable-variables conductivity function (46) can be positively employed for numerically approaching solutions of this boundary value problem, because even the magnitudes of the errors $\mathscr{E}$ are considerable bigger than those obtained when employing the original $\sigma$, their magnitudes are still acceptable when compared with other classical numerical methods.

More precisely, employing a standard finite element method technique for solving forward Dirichlet boundary value problems of elliptic partial differential equations in the plane, when considering the exponential conductivity $\sigma=$ $e^{x+y}$ and imposing the boundary condition $\left.u\right|_{\Gamma}=e^{-x-y}$, the error was $\mathscr{E}=6.1638 \times 10^{-4}$, utilizing 8257 nodes in the mesh, corresponding to 16256 triangular elements. For the Lorentzian conductivity $\sigma=\left(x^{2}+0.1\right)^{-1}\left(y^{2}+0.1\right)^{-1}$, with the boundary condition $\left.u\right|_{\Gamma}=(1 / 3)\left(x^{3}+y^{3}\right)+0.1(x+$ $y)$, employing identical number of nodes in the mesh, the resulting error was $\mathscr{E}=2.6424 \times 10^{-4}$.

A more detailed description of the behavior of the method is provided in Tables 5, 6, and 7.

Notice that the comparison with the finite element method is given as a basic reference. Indeed, the number of nodes in the mesh was taken at 8257 , because it is the closest value to the number of points located in $\Omega$ obtained when we multiply $P \cdot R=10000$.

\section{Analysis of Conductivity Functions That Are Not Separable Variables}

This section is fully dedicated to study conductivity functions that do not possess a separable-variables form, but for which we are able to obtain exact solutions of (1), in order to impose them as boundary conditions.

\subsection{The Nonseparable-Variables Exponential Case}

Proposition 14. Let the conductivity function

$$
\sigma=e^{x y}
$$

Then, a particular solution of (1) will be

$$
u=e^{-x y} \text {. }
$$

Figure 3 illustrates the conductivity funcion of (50).

Tables 7 and 8 contain the information about the behavior of the error $\mathscr{E}$ when changing the values of $S, C$, and $N$. In this case the diminution of the number of sections $S$ 
TABLE 4: Relation between the error $\mathscr{E}$ and the number of formal powers $N$, when $\sigma=e^{x+y}$.

\begin{tabular}{|c|c|c|c|c|c|}
\hline $\begin{array}{l}\text { Number of } \\
\text { radii } R\end{array}$ & $\begin{array}{l}\text { Points per } \\
\text { radius } P\end{array}$ & $\begin{array}{c}\text { Number of formal } \\
\text { powers } N\end{array}$ & $\begin{array}{c}\text { Number of } \\
\text { sections } S\end{array}$ & $\begin{array}{c}\text { Collected values per } \\
\text { section } C\end{array}$ & $\mathscr{E}$ \\
\hline 100 & 100 & 45 & 10001 & 10001 & $6.9358 \times 10^{-5}$ \\
\hline 100 & 100 & 35 & 10001 & 10001 & $4.0388 \times 10^{-4}$ \\
\hline 100 & 100 & 25 & 10001 & 10001 & $5.3651 \times 10^{-4}$ \\
\hline 100 & 100 & 15 & 10001 & 10001 & $3.4733 \times 10^{-3}$ \\
\hline 100 & 100 & 5 & 10001 & 10001 & $4.5714 \times 10^{-2}$ \\
\hline
\end{tabular}

TABLE 5: Relation between the error $\mathscr{E}$ and the number of sections $S$ and samples per section $C$, when $\sigma=\left(x^{2}+0.1\right)^{-1}\left(y^{2}+0.1\right)^{-1}$.

\begin{tabular}{|c|c|c|c|c|c|}
\hline $\begin{array}{l}\text { Number of } \\
\text { radii } R\end{array}$ & $\begin{array}{l}\text { Points per } \\
\text { radius } P\end{array}$ & $\begin{array}{l}\text { Number of formal } \\
\text { powers } N\end{array}$ & $\begin{array}{l}\text { Number of } \\
\text { sections } S\end{array}$ & $\begin{array}{c}\text { Collected values per } \\
\text { section } C\end{array}$ & $\mathscr{E}$ \\
\hline 100 & 100 & 45 & 20001 & 20001 & $6.1214 \times 10^{-6}$ \\
\hline 100 & 100 & 45 & 10001 & 10001 & $1.1241 \times 10^{-5}$ \\
\hline 100 & 100 & 45 & 5001 & 5001 & $1.3062 \times 10^{-5}$ \\
\hline 100 & 100 & 45 & 2501 & 2501 & $7.2332 \times 10^{-5}$ \\
\hline 100 & 100 & 45 & 1001 & 1001 & $1.7079 \times 10^{-4}$ \\
\hline 100 & 100 & 45 & 501 & 501 & $3.5471 \times 10^{-4}$ \\
\hline 100 & 100 & 45 & 101 & 101 & $1.0553 \times 10^{-3}$ \\
\hline
\end{tabular}

TABLE 6: Relation between the error $\mathscr{E}$ and the number of formal powers $N$, when $\sigma=\left(x^{2}+0.1\right)^{-1}\left(y^{2}+0.1\right)^{-1}$.

\begin{tabular}{|c|c|c|c|c|c|}
\hline $\begin{array}{l}\text { Number of } \\
\text { radii } R\end{array}$ & $\begin{array}{c}\text { Points per } \\
\text { radius } P\end{array}$ & $\begin{array}{c}\text { Number of formal } \\
\text { powers } N\end{array}$ & $\begin{array}{l}\text { Number of } \\
\text { sections } S\end{array}$ & $\begin{array}{l}\text { Collected values per } \\
\text { section } C\end{array}$ & $\mathscr{E}$ \\
\hline 100 & 100 & 45 & 10001 & 10001 & $1.1241 \times 10^{-5}$ \\
\hline 100 & 100 & 35 & 10001 & 10001 & $9.1964 \times 10^{-5}$ \\
\hline 100 & 100 & 25 & 10001 & 10001 & $5.0855 \times 10^{-4}$ \\
\hline 100 & 100 & 15 & 10001 & 10001 & $4.2633 \times 10^{-3}$ \\
\hline 100 & 100 & 5 & 10001 & 10001 & $2.9065 \times 10^{-2}$ \\
\hline
\end{tabular}

TABLE 7: Relation between the error $\mathscr{E}$ and the number of sections $S$ and samples per section $C$, when $\sigma=e^{x y}$.

\begin{tabular}{|c|c|c|c|c|c|}
\hline $\begin{array}{l}\text { Number of } \\
\text { radii } R\end{array}$ & $\begin{array}{l}\text { Points per } \\
\text { radius } P \\
\end{array}$ & $\begin{array}{c}\text { Number of formal } \\
\text { powers } N\end{array}$ & $\begin{array}{c}\text { Number of } \\
\text { sections } S\end{array}$ & $\begin{array}{c}\text { Collected values per } \\
\text { section } C \\
\end{array}$ & $\mathscr{E}$ \\
\hline 100 & 100 & 45 & 20001 & 20001 & $3.8759 \times 10^{-6}$ \\
\hline 100 & 100 & 45 & 10001 & 10001 & $5.4177 \times 10^{-6}$ \\
\hline 100 & 100 & 45 & 5001 & 5001 & $2.1470 \times 10^{-5}$ \\
\hline 100 & 100 & 45 & 2501 & 2501 & $1.5406 \times 10^{-5}$ \\
\hline 100 & 100 & 45 & 1001 & 1001 & $6.6917 \times 10^{-5}$ \\
\hline 100 & 100 & 45 & 501 & 501 & $1.5059 \times 10^{-4}$ \\
\hline 100 & 100 & 45 & 101 & 101 & $8.7576 \times 10^{-4}$ \\
\hline
\end{tabular}

TABLE 8: Relation between the error $\mathscr{E}$ and the number of formal powers $N$, when $\sigma=e^{x y}$.

\begin{tabular}{|c|c|c|c|c|c|}
\hline $\begin{array}{l}\text { Number of } \\
\text { radii } R\end{array}$ & $\begin{array}{l}\text { Points per } \\
\text { radius } P\end{array}$ & $\begin{array}{c}\text { Number of formal } \\
\text { powers } N\end{array}$ & $\begin{array}{l}\text { Number of } \\
\text { sections } S\end{array}$ & $\begin{array}{l}\text { Collected values per } \\
\text { section } C\end{array}$ & $\mathscr{E}$ \\
\hline 100 & 100 & 45 & 10001 & 10001 & $5.4177 \times 10^{-6}$ \\
\hline 100 & 100 & 35 & 10001 & 10001 & $2.6425 \times 10^{-5}$ \\
\hline 100 & 100 & 25 & 10001 & 10001 & $3.2504 \times 10^{-5}$ \\
\hline 100 & 100 & 15 & 10001 & 10001 & $3.4497 \times 10^{-5}$ \\
\hline 100 & 100 & 5 & 10001 & 10001 & $1.0798 \times 10^{-2}$ \\
\hline
\end{tabular}


TABLE 9: Relation between the error $\mathscr{E}$ and the number of sections $S$ and samples per section $C$, when $\sigma=\left((x+y)^{2}+1\right)^{-1}$.

\begin{tabular}{|c|c|c|c|c|c|}
\hline $\begin{array}{l}\text { Number of } \\
\text { radii } R\end{array}$ & $\begin{array}{l}\text { Points per } \\
\text { radius } P\end{array}$ & $\begin{array}{c}\text { Number of formal } \\
\text { powers } N\end{array}$ & $\begin{array}{c}\text { Number of } \\
\text { sections } S\end{array}$ & $\begin{array}{c}\text { Collected values per } \\
\text { section } C\end{array}$ & $\mathscr{E}$ \\
\hline 100 & 100 & 45 & 20001 & 20001 & $8.8364 \times 10^{-3}$ \\
\hline 100 & 100 & 45 & 10001 & 10001 & $8.9246 \times 10^{-3}$ \\
\hline 100 & 100 & 45 & 5001 & 5001 & $9.4573 \times 10^{-3}$ \\
\hline 100 & 100 & 45 & 2501 & 2501 & $9.9164 \times 10^{-3}$ \\
\hline 100 & 100 & 45 & 1001 & 1001 & $1.0330 \times 10^{-2}$ \\
\hline 100 & 100 & 45 & 501 & 501 & $8.6507 \times 10^{-3}$ \\
\hline 100 & 100 & 45 & 101 & 101 & $9.5800 \times 10^{-3}$ \\
\hline
\end{tabular}

TABLE 10: Relation between the error $\mathscr{E}$ and the number of formal powers $N$, when $\sigma=\left((x+y)^{2}+1\right)^{-1}$.

\begin{tabular}{|c|c|c|c|c|c|}
\hline $\begin{array}{l}\text { Number of } \\
\text { radii } R\end{array}$ & $\begin{array}{l}\text { Points per } \\
\text { radius } P\end{array}$ & $\begin{array}{l}\text { Number of formal } \\
\text { powers } N\end{array}$ & $\begin{array}{l}\text { Number of } \\
\text { sections } S\end{array}$ & $\begin{array}{c}\text { Collected values per } \\
\text { section } C\end{array}$ & $\mathscr{E}$ \\
\hline 100 & 100 & 45 & 10001 & 10001 & $8.9246 \times 10^{-3}$ \\
\hline 100 & 100 & 35 & 10001 & 10001 & $4.3227 \times 10^{-2}$ \\
\hline 100 & 100 & 25 & 10001 & 10001 & $5.9625 \times 10^{-2}$ \\
\hline 100 & 100 & 15 & 10001 & 10001 & $8.2125 \times 10^{-2}$ \\
\hline 100 & 100 & 5 & 10001 & 10001 & $1.4064 \times 10^{-1}$ \\
\hline
\end{tabular}

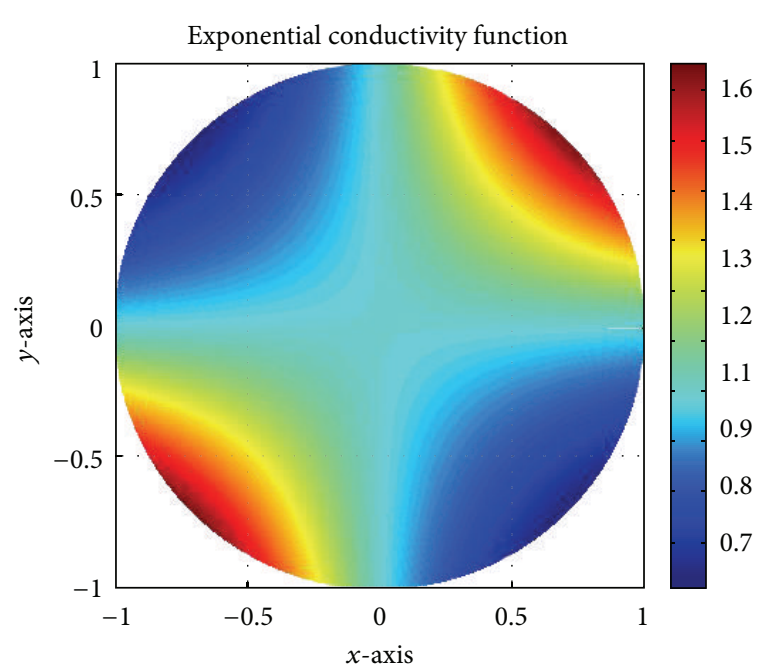

FIGURE 3: Example of nonseparable-variables conductivity function $\sigma=e^{x y}$.

and diminution of the samples per section $C$ do influence the accuracy, but their influence is not so significant as the diminution of the formal powers $N$.

The error obtained when employing the finite element method approach was $\mathscr{E}=2.3966 \times 10^{-4}$, employing 8257 points in the mesh.

\subsection{The Nonseparable-Variables Lorentzian Case}

Proposition 15. Let the conductivity function have the form

$$
\sigma=\frac{1}{(x+y)^{2}+1}
$$

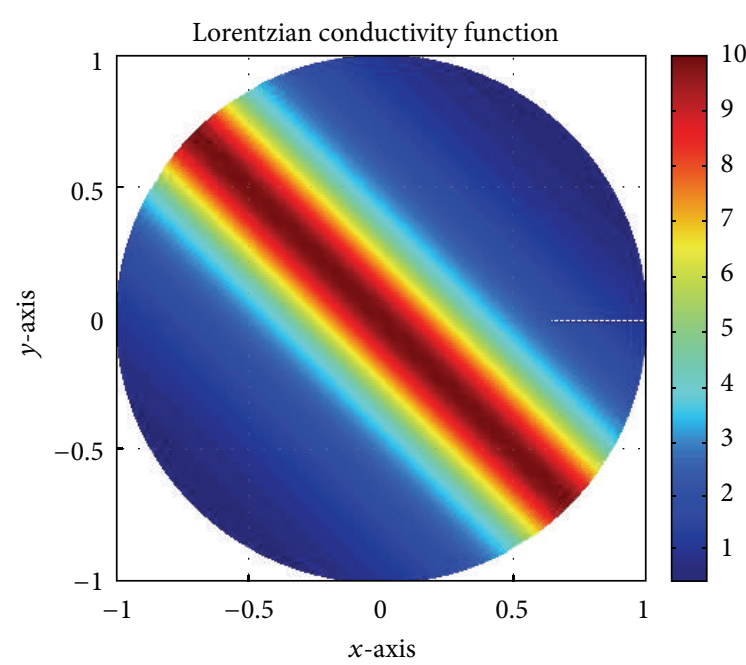

FIGURE 4: Example of nonseparable-variables Lorentzian conductivity function $\sigma=\left((x+y)^{2}+1\right)^{-1}$.

An exact solution for (1) is

$$
u=\frac{(x+y)^{3}}{3}+x+y .
$$

The conductivity function with the form (52) is illustrated in Figure 4.

Table 9 shows an abnormal behavior of the error $\mathscr{E}$ when decreasing the number of sections $S$ and samples $C$. That is, it does not follow a clear pattern. But more important is to remark that the finite element method reported an error $\mathscr{E}=5.4311 \times 10^{-4}$, which implies that is better situated for analyzing this class of conductivity functions. Table 10 


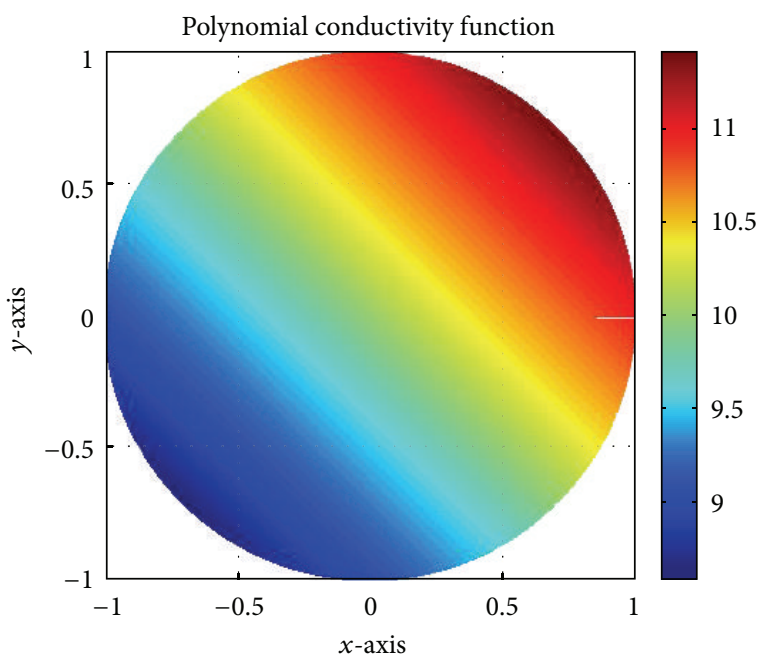

FIGURE 5: Examples of nonseparable-variables polynomial conductivity function $\sigma=x+y+10$.

states that the diminution of the number of formal powers $N$ decreases the accuracy.

\subsection{The Nonseparable-Variables Polynomial Case}

Proposition 16. Let one assume that the conductivity function has the form

$$
\sigma=x+y+10
$$

thus the function

$$
u=\ln (x+y+10)
$$

will be a solution of (1).

In Figure 5 it is illustrated the polynomial conductivity function of (54).

Tables 11 and 12 show a regular behavior of the error $\mathscr{E}$, because it decreases when the values $S$ and $C$ do. This case possesses a singular characteristic when compared to the cases above: The diminution of the number of formal powers $N$ does not provoke a considerable loss of the accuracy. This exception will be remarked since it has not been detected in other class of conductivity functions.

On the other hand, the finite element method proved to provide a very similar accuracy for analyzing this case, because the obtained error was $\mathscr{E}=2.8958 \times 10^{-6}$.

\subsection{The Nonseparable-Variables Sinusoidal Case}

Proposition 17. Let one consider the sinusoidal conductivity

$$
\sigma=2+\sin (x+y) \text {. }
$$

One can verify by direct substitution that the function

$$
u=\frac{2}{\sqrt{3}} \arctan \left(\frac{2 \tan ((x+y) / 2)+1}{\sqrt{3}}\right)
$$

is a solution of (1).
The Figure 6(a) illustrates the conductivity (56), whereas the Figure 6(b) illustrates the case (58).

Tables 13 and 14 show that the behavior of $\mathscr{E}$ is not fully regular, because a diminution of the number $S$ and $C$ does not necessarily imply a reduction of the accuracy, whereas the diminution of the formal powers $N$ provokes significant changes of the error $\mathscr{E}$. For this case, the finite element method reported an error $\mathscr{E}=0.0192$.

Notice that the exact solution (57) that was imposed as the boundary condition $\left.u\right|_{\Gamma}$ cannot be employed when a constant factor multiplies the argument of the trigonometric function, because the tangent would not be defined at certain points. Nevertheless, one last experiment is worth of research.

Let us suppose that the conductivity function possesses the form

$$
\sigma=2+\sin 5(x+y)
$$

and let us approach the solution to the forward problem keeping as the boundary condition the expression (57). Tables 15 and 16 display the biggest error $\mathscr{E}$ found in the present work, even the behavior of $\mathscr{E}$ could be considered regular, because it decreases while $S, C$, and $N$ do. The reader will appreciate that the accuracy is, in general, unsatisfactory for applications. Indeed, the finite element method obtained an error $\mathscr{E}=0.0043$, hence it is quite better situated for analyzing this kind of conductivity functions.

\section{Conclusions}

The comparison of the numerical method posed in this work and the finite element method is far to be complete. Thus the selected examples will be considered a basic reference from which many other trials must be adequately performed, since they could not be included in these pages because they constitute independent research topics. For instance, a detailed description of the computational complexity [12] of both methods would provide a point of view less dependent on the platform on which the methods are programmed; thus we could focus our attention into the basic properties.

Still, we will remark an important characteristic of the pseudoanalytic functions-based method. It provides information about the solutions of the forward Dirichlet boundary value problem for (1), that is not clear how to obtain when utilizing the finite element method.

More precisely, the numerical approach based upon the pseudoanalytic analysis reaches a full set of functions defined at the boundary, that might contain relevant data if we desire to examine the electrical impedance tomography problem.

For instance, let us consider the base functions $\left\{u_{n}\right\}_{n=0}^{2 N+1}$ approached for the case when $\sigma=e^{x y}$, as examined in (50). The absolute values of the coefficients $\alpha_{n}$, employed for approaching the boundary condition $\left.u\right|_{\Gamma}=e^{-x y}$, are displayed in the semilogarithmic graphic of Figure 7 . This calculation was performed considering $S=20001$ sections, $C=10001$ samples per section, $R=100$ radii, $P=101$ points per radii, and $N=45$ formal powers; thus we will have $2 N+1=91$ base functions $u_{n}$, defined at the boundary $\Gamma$. 


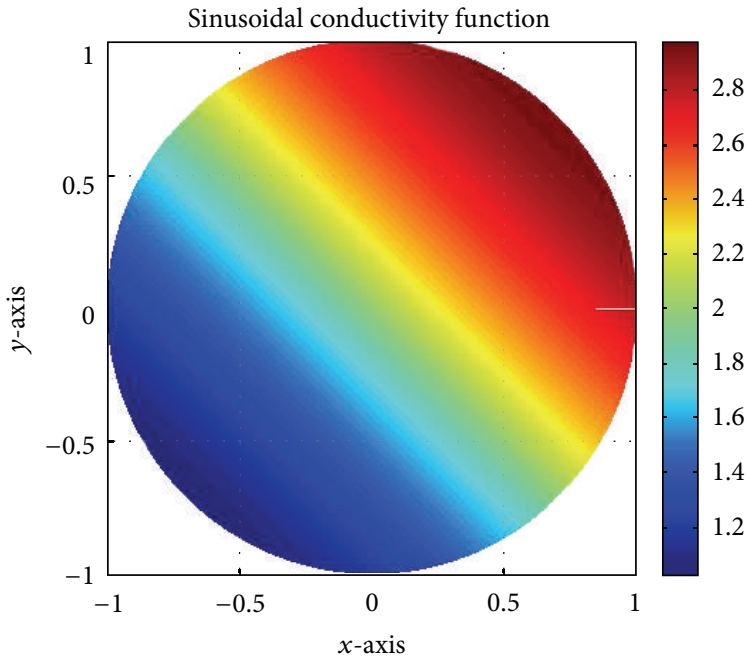

(a) $\sigma=2+\sin (x+y)$

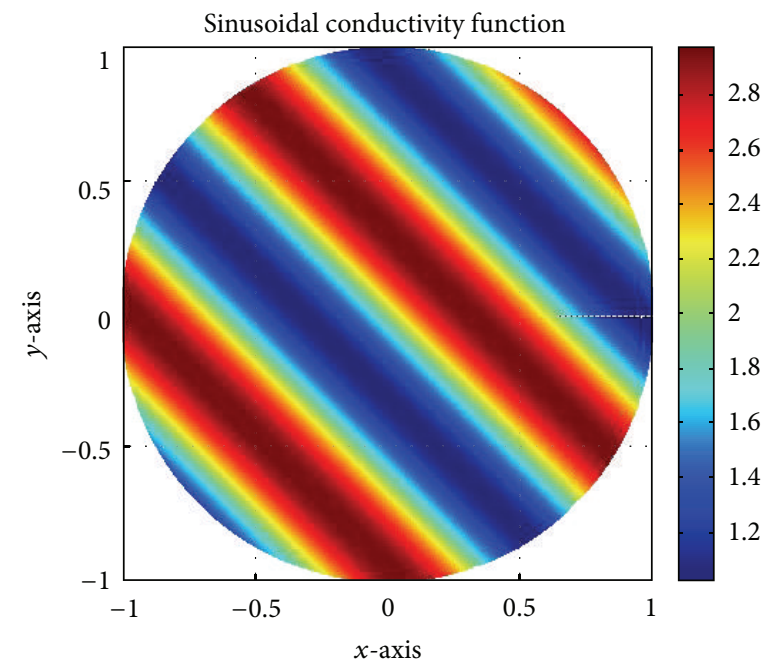

(b) $\sigma=2+\sin 5(x+y)$

FIGURE 6: Examples of sinusoidal conductivity functions.

TABLE 11: Relation between the error $\mathscr{E}$ and the number of sections $S$ and samples per section $C$, when $\sigma=x+y+10$.

\begin{tabular}{|c|c|c|c|c|c|}
\hline $\begin{array}{l}\text { Number of } \\
\text { radii } R\end{array}$ & $\begin{array}{l}\text { Points per } \\
\text { radius } P\end{array}$ & $\begin{array}{c}\text { Number of formal } \\
\text { powers } N\end{array}$ & $\begin{array}{c}\text { Number of } \\
\text { sections } S\end{array}$ & $\begin{array}{c}\text { Collected values per } \\
\text { section } C\end{array}$ & $\mathscr{E}$ \\
\hline 100 & 100 & 45 & 20001 & 20001 & $3.0640 \times 10^{-6}$ \\
\hline 100 & 100 & 45 & 10001 & 10001 & $2.3420 \times 10^{-6}$ \\
\hline 100 & 100 & 45 & 5001 & 5001 & $8.1962 \times 10^{-6}$ \\
\hline 100 & 100 & 45 & 2501 & 2501 & $1.9286 \times 10^{-5}$ \\
\hline 100 & 100 & 45 & 1001 & 1001 & $6.4916 \times 10^{-5}$ \\
\hline 100 & 100 & 45 & 501 & 501 & $1.5166 \times 10^{-4}$ \\
\hline 100 & 100 & 45 & 101 & 101 & $4.5938 \times 10^{-4}$ \\
\hline
\end{tabular}

TABLE 12: Relation between the error $\mathscr{E}$ and the number of formal powers $N$, when $\sigma=x+y+10$.

\begin{tabular}{|c|c|c|c|c|c|}
\hline $\begin{array}{l}\text { Number of } \\
\text { radii } R\end{array}$ & $\begin{array}{l}\text { Points per } \\
\text { radius } P\end{array}$ & $\begin{array}{c}\text { Number of formal } \\
\text { powers } N\end{array}$ & $\begin{array}{l}\text { Number of } \\
\text { sections } S\end{array}$ & $\begin{array}{c}\text { Collected values per } \\
\text { section } C\end{array}$ & $\mathscr{E}$ \\
\hline 100 & 100 & 45 & 10001 & 10001 & $2.3420 \times 10^{-6}$ \\
\hline 100 & 100 & 35 & 10001 & 10001 & $1.0616 \times 10^{-5}$ \\
\hline 100 & 100 & 25 & 10001 & 10001 & $1.6275 \times 10^{-5}$ \\
\hline 100 & 100 & 15 & 10001 & 10001 & $1.8396 \times 10^{-5}$ \\
\hline 100 & 100 & 5 & 10001 & 10001 & $1.9909 \times 10^{-5}$ \\
\hline
\end{tabular}

TABLE 13: Relation between the error $\mathscr{E}$ and the number of sections $S$ and samples per section $C$, when $\sigma=2+\sin (x+y)$.

\begin{tabular}{|c|c|c|c|c|c|}
\hline $\begin{array}{l}\text { Number of } \\
\text { radii } R\end{array}$ & $\begin{array}{l}\text { Points per } \\
\text { radius } P\end{array}$ & $\begin{array}{c}\text { Number of formal } \\
\text { powers } N\end{array}$ & $\begin{array}{l}\text { Number of } \\
\text { sections } S\end{array}$ & $\begin{array}{c}\text { Collected values per } \\
\text { section } C\end{array}$ & $\mathscr{E}$ \\
\hline 100 & 100 & 45 & 20001 & 20001 & $7.5333 \times 10^{-2}$ \\
\hline 100 & 100 & 45 & 10001 & 10001 & $7.6129 \times 10^{-2}$ \\
\hline 100 & 100 & 45 & 5001 & 5001 & $7.4343 \times 10^{-2}$ \\
\hline 100 & 100 & 45 & 2501 & 2501 & $6.9999 \times 10^{-2}$ \\
\hline 100 & 100 & 45 & 1001 & 1001 & $8.4363 \times 10^{-2}$ \\
\hline 100 & 100 & 45 & 501 & 501 & $6.4758 \times 10^{-2}$ \\
\hline 100 & 100 & 45 & 101 & 101 & $9.0737 \times 10^{-2}$ \\
\hline
\end{tabular}


TABLE 14: Relation between the error $\mathscr{E}$ and the number of formal powers $N$, when $\sigma=2+\sin (x+y)$.

\begin{tabular}{|c|c|c|c|c|c|}
\hline $\begin{array}{l}\text { Number of } \\
\text { radii } R\end{array}$ & $\begin{array}{l}\text { Points per } \\
\text { radius } P\end{array}$ & $\begin{array}{c}\text { Number of formal } \\
\text { powers } N\end{array}$ & $\begin{array}{l}\text { Number of } \\
\text { sections } S\end{array}$ & $\begin{array}{l}\text { Collected values per } \\
\text { section } C\end{array}$ & $\mathscr{E}$ \\
\hline 100 & 100 & 45 & 10001 & 10001 & $7.6129 \times 10^{-2}$ \\
\hline 100 & 100 & 35 & 10001 & 10001 & $1.3551 \times 10^{-1}$ \\
\hline 100 & 100 & 25 & 10001 & 10001 & $2.0886 \times 10^{-1}$ \\
\hline 100 & 100 & 15 & 10001 & 10001 & $3.3854 \times 10^{-1}$ \\
\hline 100 & 100 & 5 & 10001 & 10001 & $5.3026 \times 10^{-1}$ \\
\hline
\end{tabular}

TABLE 15: Relation between the error $\mathscr{E}$ and the number of sections $S$ and samples per section $C$, when $\sigma=2+\sin 5(x+y)$.

\begin{tabular}{|c|c|c|c|c|c|}
\hline $\begin{array}{l}\text { Number of } \\
\text { radii } R\end{array}$ & $\begin{array}{c}\text { Points per } \\
\text { radius } P\end{array}$ & $\begin{array}{c}\text { Number of formal } \\
\text { powers } N\end{array}$ & $\begin{array}{c}\text { Number of } \\
\text { sections } S \\
\end{array}$ & $\begin{array}{c}\text { Collected values per } \\
\text { section } C\end{array}$ & $\mathscr{E}$ \\
\hline 100 & 100 & 45 & 20001 & 20001 & $7.5333 \times 10^{-2}$ \\
\hline 100 & 100 & 45 & 10001 & 10001 & $7.6129 \times 10^{-2}$ \\
\hline 100 & 100 & 45 & 5001 & 5001 & $7.4343 \times 10^{-2}$ \\
\hline 100 & 100 & 45 & 2501 & 2501 & $6.9999 \times 10^{-2}$ \\
\hline 100 & 100 & 45 & 1001 & 1001 & $8.4363 \times 10^{-2}$ \\
\hline 100 & 100 & 45 & 501 & 501 & $6.4758 \times 10^{-2}$ \\
\hline 100 & 100 & 45 & 101 & 101 & $9.0737 \times 10^{-2}$ \\
\hline
\end{tabular}

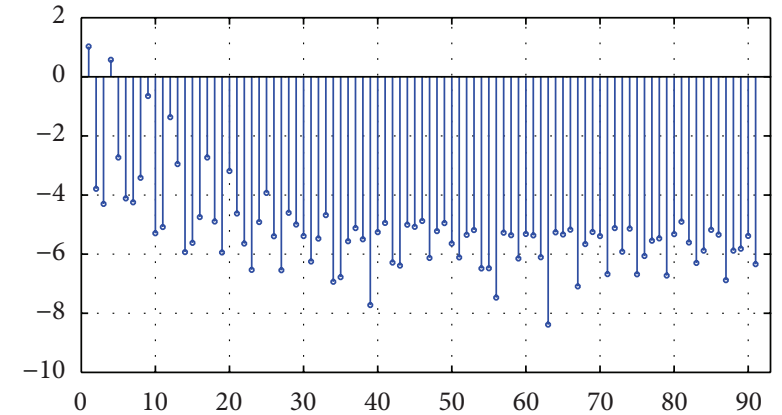

Figure 7: Absolute values of the coefficients $\alpha_{n}$ employed for approaching the boundary condition $\left.u\right|_{\Gamma}=e^{-x y}$, utilizing the base elements $\left\{u_{n}\right\}$ corresponding to the conductivity function $\sigma=e^{x y}$.

Notice that in Figure 7 the values of the coefficients $\alpha_{n}$ possess a particular distribution, even we will not point an evident pattern. But since the set $\left\{u_{n}\right\}_{n=0}^{2 N+1}$ is an orthonormal base, it can be used to approach any other boundary condition. In particular, if we use this base for approaching the condition

$$
\left.u\right|_{\Gamma}=\frac{1}{3}(x+y)^{3}+0.1(x+y),
$$

that indeed corresponds to the conductivity $\sigma=\left((x+y)^{2}+\right.$ $0.1)^{-1}$, we will observe that the values of the coefficients $\alpha_{n}$, plotted in Figure 8, behave in a very different way.

As a complementary example, let us consider the base functions $\left\{u_{n}\right\}$ corresponding to a Lorentzian conductivity $\sigma=\left((x+y)^{2}+0.1\right)^{-1}$ studied in Section 4.2. Figure 9 displays the absolute values of the coefficients $\alpha_{n}$ when we impose the boundary condition $\left.u\right|_{\Gamma}=(1 / 3)(x+y)^{3}+0.1(x+$ $y)$, as posed in (53).

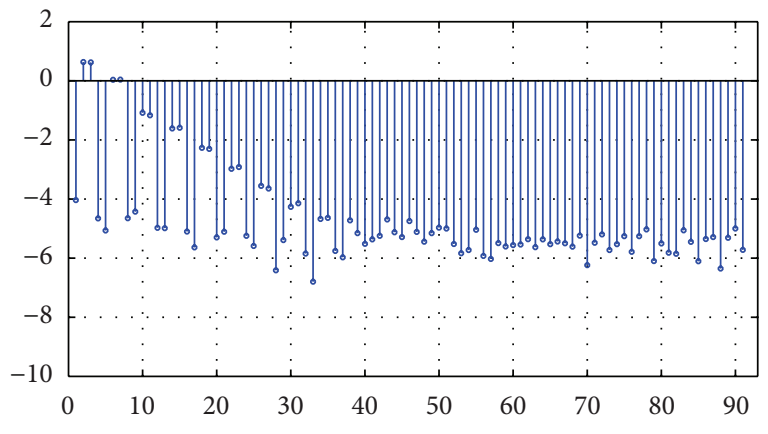

FIGURE 8: Absolute values of the coefficients $\alpha_{n}$ employed for approaching the boundary condition $\left.u\right|_{\Gamma}=(1 / 3)(x+y)^{3}+0.1(x+$ $y$ ), utilizing the base elements $\left\{u_{n}\right\}$ corresponding to the conductivity function $\sigma=e^{x y}$.

But if we impose a boundary condition of the form

$$
\left.u\right|_{\Gamma}=e^{-x y},
$$

upcoming from (51), we can appreciate, as plotted in Figure 10, that the absolute values of the obtained coefficients $\alpha_{n}$ are, once more, very different to those of Figure 9.

This implies that the behavior of the values of the coefficients $\alpha_{n}$ could well serve as a criterion for understanding if some conductivity function $\sigma$ can effectively provoke a certain electric potential distribution at the boundary $\Gamma$ of same domain $\Omega\left(\mathbb{R}^{2}\right)$, when physical measurements provide the boundary condition.

Taking into account that most of the algorithms dedicated to approach solutions of the electrical impedance tomography problem recursively solve the forward problem [2], this novel information will be used to introduce changes in $\sigma$; thus the boundary condition is fulfilled with a better convergence, and with less computational time. 
TABLE 16: Relation between the error $\mathscr{E}$ and the number of formal powers $N$, when $\sigma=2+\sin 5(x+y)$.

\begin{tabular}{lccccc}
\hline $\begin{array}{l}\text { Number of } \\
\text { radii } R\end{array}$ & $\begin{array}{c}\text { Points per } \\
\text { radius } P\end{array}$ & $\begin{array}{c}\text { Number of formal } \\
\text { powers } N\end{array}$ & $\begin{array}{c}\text { Number of } \\
\text { sections } S\end{array}$ & $\begin{array}{c}\text { Collected values per } \\
\text { section } C\end{array}$ & $\mathscr{E}$ \\
\hline 100 & 100 & 45 & 10001 & 10001 & $7.6129 \times 10^{-2}$ \\
100 & 100 & 35 & 10001 & 10001 & $1.3551 \times 10^{-1}$ \\
100 & 100 & 25 & 10001 & 10001 & $2.0886 \times 10^{-1}$ \\
100 & 100 & 15 & 10001 & 10001 & $3.3854 \times 10^{-1}$ \\
100 & 100 & 5 & 10001 & 10001 & $5.3026 \times 10^{-1}$ \\
\hline
\end{tabular}

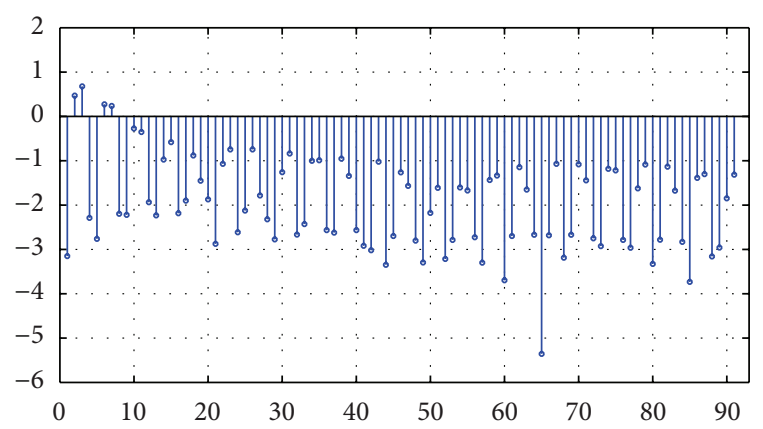

Figure 9: Absolute values of the coefficients $\alpha_{n}$ employed for approaching the boundary condition $\left.u\right|_{\Gamma}=(1 / 3)(x+y)^{3}+0.1(x+$ $y)$, utilizing the base elements $\left\{u_{n}\right\}$ corresponding to the conductivity function $\sigma=\left((x+y)^{2}+0.1\right)^{-1}$.

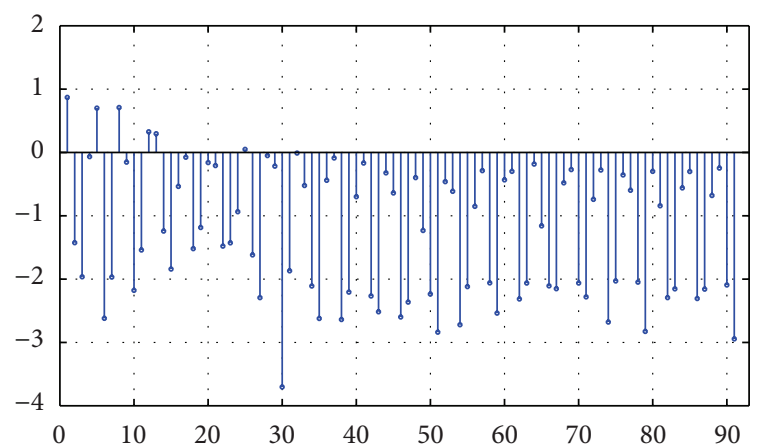

Figure 10: Absolute values of the coefficients $\alpha_{n}$ employed for approaching the boundary condition $\left.u\right|_{\Gamma}=e^{-x y}$, utilizing the base elements $\left\{u_{n}\right\}$ corresponding to the conductivity function $\sigma=$ $\left((x+y)^{2}+0.1\right)^{-1}$.

Even it is not possible yet to estimate when this new method will be employed to approach solutions for the electrical impedance tomography problem, we will remark that the main contribution of this work is to provide a new technique for fully applying the elements of the modern Pseudoanalytic function theory into the analysis of conductivity functions that originated from physical experimental models, which will certainly provide additional information for better understanding the inverse Dirichlet boundary value problem of (1) in the plane.
In other words, before this proposal, the mathematical advances were exclusively improving the study of separablevariables conductivity functions, rarely useful for representing physical cases. These pages have shown that, under certain limits, any conductivity function is susceptible to be analyzed employing pseudoanalytic functions, whether they possess an exact mathematical form or not. As a matter of fact, it is enough that the conductivity values are fully defined over the points within a bounded domain $\Omega$, with a smooth enough boundary $\Gamma$. The research will continue to better understand the limiting cases and the certain conditions of smoothness of the boundary; thus the posed ideas can be adequately employed in the applied sciences.

\section{Disclosure}

The numerical methods used along this work were fully developed in GNU C/C++ Compiler, employing a CPU64B@ 2.4 GHz, on SLACKWARE 13.37 LINUX operating system. The experimental procedures showed that the numerical results can vary when using different platforms based on $32 \mathrm{~B}$ and $64 \mathrm{~B}$ processor architecture, or compilers between other operating systems, including different LINUX distributions or Registered Trade Mark operating systems. If the reader wishes to perform his own numerical trials, please contact the authors to obtain the resource codes.

\section{Acknowledgments}

The authors would like to acknowledge the support of CONACyT Projects 106722 and 81599, Mexico. Marco Pedro Ramirez-Tachiquin is grateful for the support of HILMA S.A. de C.V., Mexico; Ariana Guadalupe Bucio Ramirez would like to acknowledge the support of CONACyT and UPIITA, IPN; Rogelio Adrian Hernandez-Becerril and Cesar Marco Antonio Robles Gonzalez would like to acknowledge the support of CONACyT and ESIME, IPN.

\section{References}

[1] A.-P. Calderon, "On an inverse boundary value problem," in Seminar on Numerical Analysis and Its Applications to Continuum Physics, pp. 65-73, Sociedade Brasileira de Matematica, Rio de Janeiro, Brazil, 1980.

[2] J. G. Webster, Electrical Impedance Tomography, Adam Hilger Series on Biomedical Engineering, Adam Hilger, Briston, UK, 1990. 
[3] V. Kravchenko, "On a relation of pseudoanalytic function theory to the two-dimensional stationary Schrödinger equation and Taylor series in formal powers for its solutions," Journal of Physics A, vol. 38, no. 18, pp. 3947-3964, 2005.

[4] K. Astala and L. Päivärinta, "Calderón's inverse conductivity problem in the plane," Annals of Mathematics, vol. 163, no. 1, pp. 265-299, 2006.

[5] I. N. Vekua, Generalized Analytic Functions, International Series of Monographs on Pure and Applied Mathematics, Pergamon Press, London, UK, 1962.

[6] V. V. Kravchenko and H. Oviedo, "On explicitly solvable Vekua equations and explicit solution of the stationary Schrödinger equation and of the equation $(\sigma \nabla u)=0$," Complex Variables and Elliptic Equations, vol. 52, no. 5, pp. 353-366, 2007.

[7] L. Bers, Theory of Pseudoanalytic Functions, IMM, New York University, New York, NY, USA, 1953.

[8] H. M. Campos, R. Castillo-Pérez, and V. V. Kravchenko, "Construction and application of Bergman-type reproducing kernels for boundary and eigenvalue problems in the plane," Complex Variables and Elliptic Equations, vol. 57, no. 7-8, pp. 787-824, 2012.

[9] V. V. Kravchenko, Applied Pseudoanalytic Function Theory, Frontiers in Mathematics, Birkhäuser, Basel, Switzerland, 2009.

[10] R. Castillo-Pérez, V. V. Kravchenko, and R. Reséndiz-Vázquez, "Solution of boundary and eigenvalue problems for secondorder elliptic operators in the plane using pseudoanalytic formal powers," Mathematical Methods in the Applied Sciences, vol. 34, no. 4, pp. 455-468, 2011.

[11] A. Bucio R, R. Castillo-Perez, and M. P. Ramirez T., "On the numerical construction of formal powers and their application to the Electrical Impedance Equation," in Proceedings of the 8th International Conference on Electrical Engineering, Computing Science and Automatic Control (CCE '11), IEEE Catalog Number: CFP11827-ART, pp. 769-774, mex, October 2011.

[12] A. Sanjeev and B. Boaz, Computational Complexity: A Modern Approach, Cambridge University Press, Cambridge, UK, 2009. 


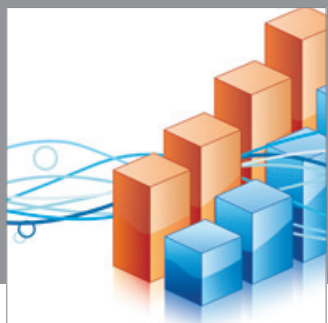

Advances in

Operations Research

mansans

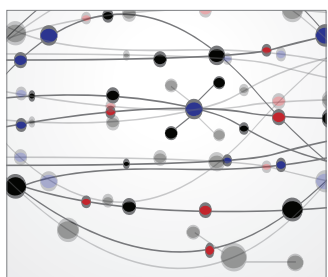

The Scientific World Journal
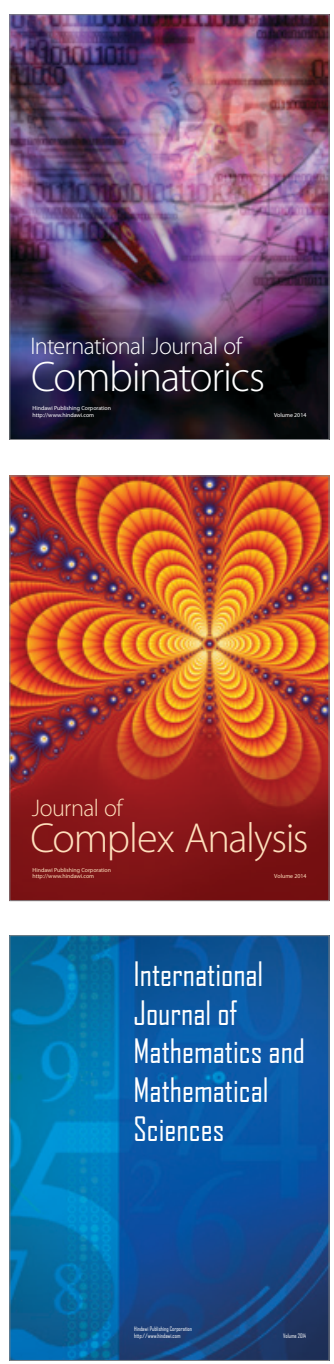
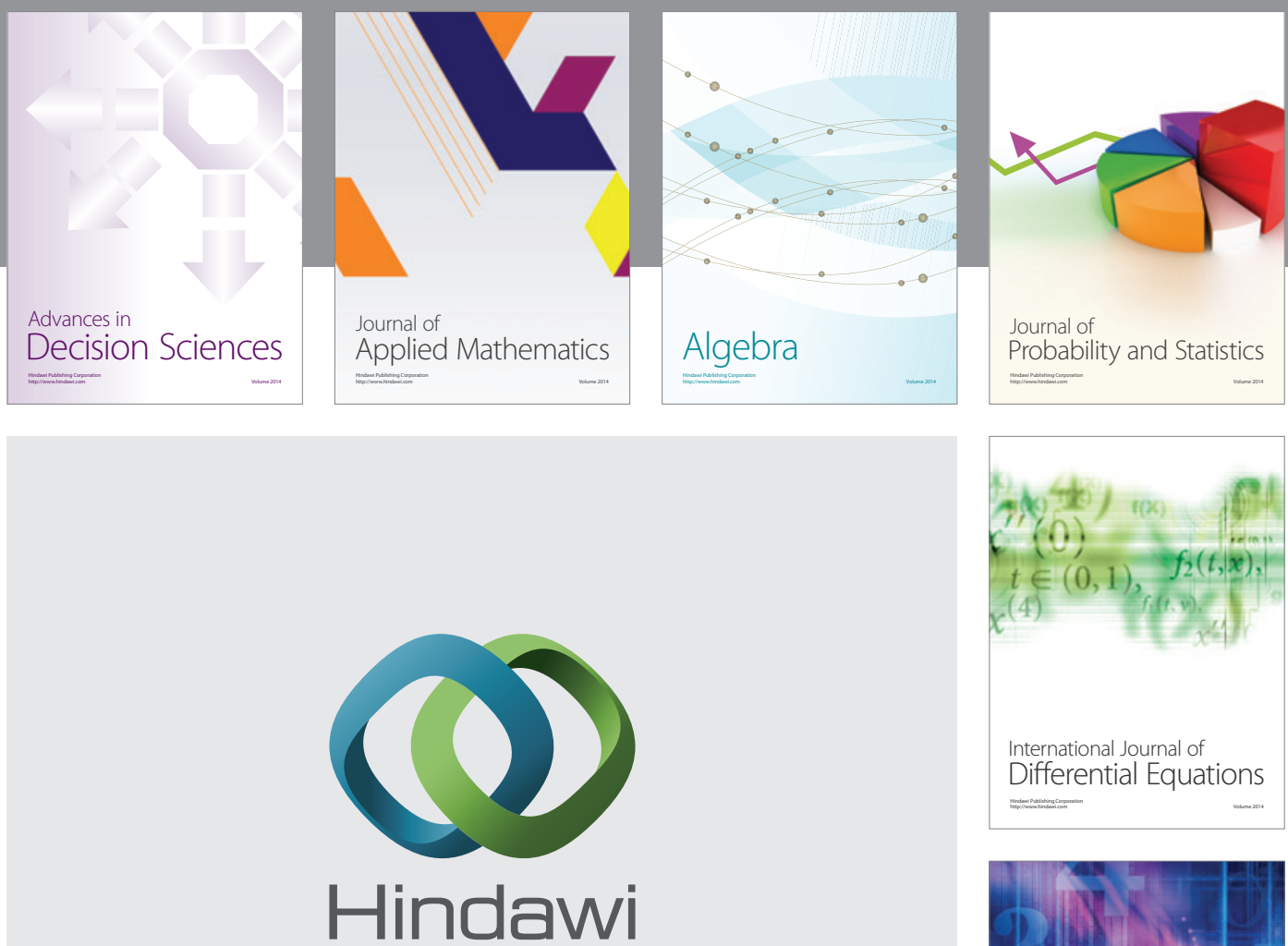

Submit your manuscripts at http://www.hindawi.com
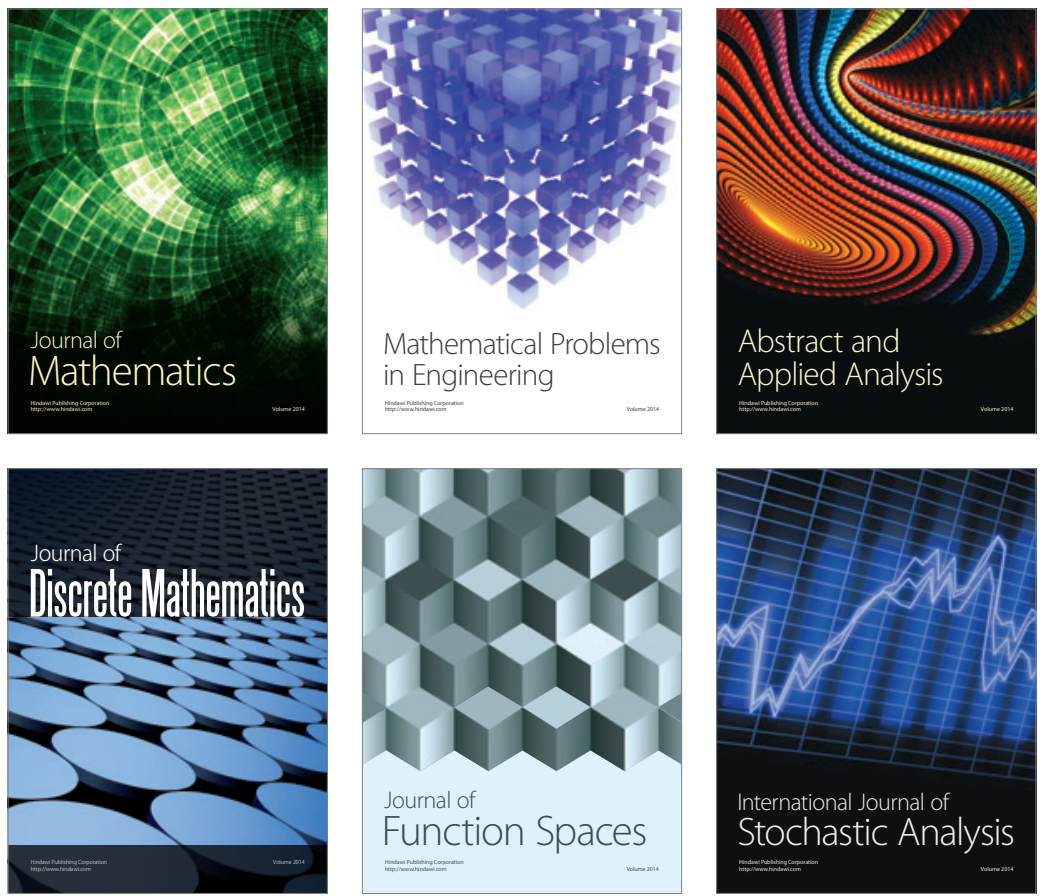

Journal of

Function Spaces

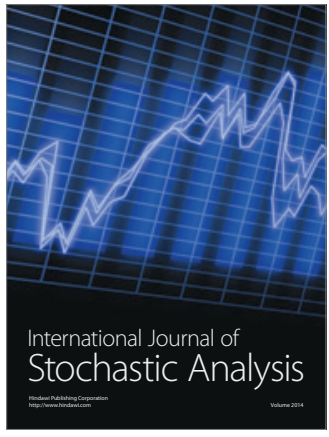

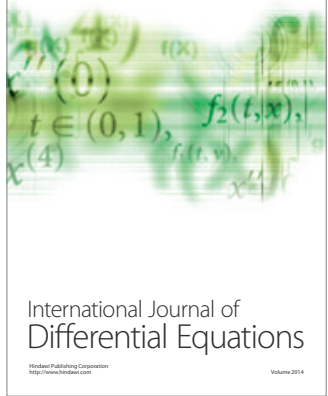
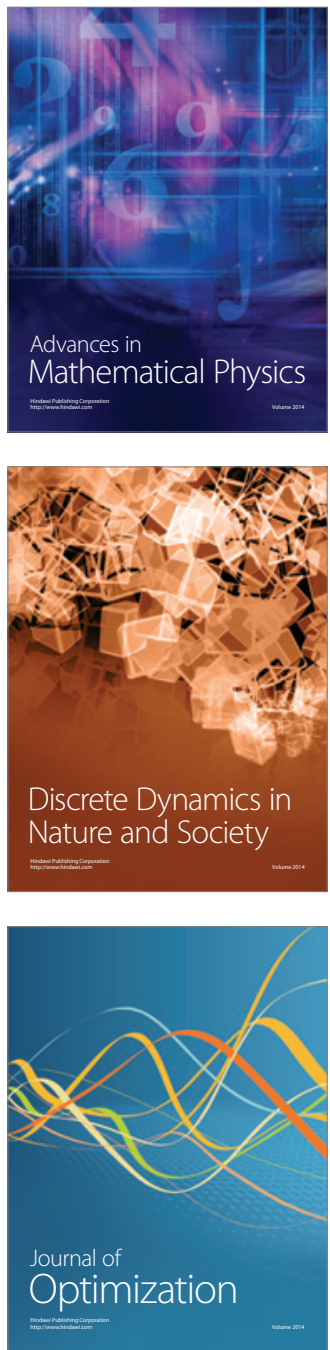